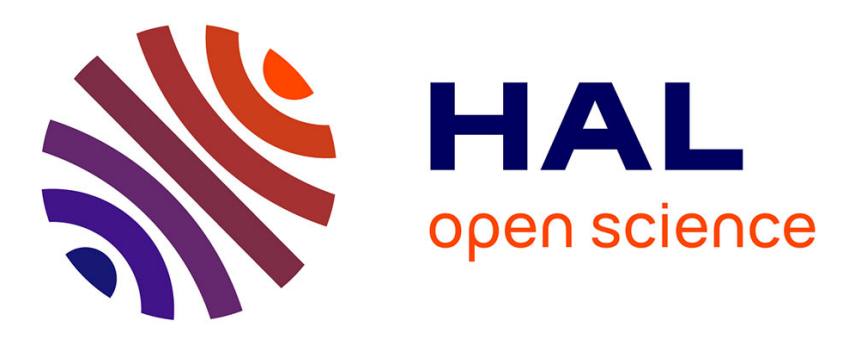

\title{
Nonlinear joint state and parameter estimation : application to a wastewater treatment plant
} Souad Bezzaoucha, Benoît Marx, Didier Maquin, José Ragot

\section{To cite this version:}

Souad Bezzaoucha, Benoît Marx, Didier Maquin, José Ragot. Nonlinear joint state and parameter estimation: application to a wastewater treatment plant. Control Engineering Practice, 2013, 21 (10), pp.1377-1385. 10.1016/j.conengprac.2013.06.009 . hal-00831876

\section{HAL Id: hal-00831876 https://hal.science/hal-00831876}

Submitted on 9 Apr 2014

HAL is a multi-disciplinary open access archive for the deposit and dissemination of scientific research documents, whether they are published or not. The documents may come from teaching and research institutions in France or abroad, or from public or private research centers.
L'archive ouverte pluridisciplinaire HAL, est destinée au dépôt et à la diffusion de documents scientifiques de niveau recherche, publiés ou non, émanant des établissements d'enseignement et de recherche français ou étrangers, des laboratoires publics ou privés. 


\title{
Nonlinear Joint State and Parameter Estimation : Application to a Wastewater Treatment Plant
}

\author{
S. Bezzaouchaa, ${ }^{\mathrm{a} b}$ B. Marx ${ }^{\mathrm{a}, \mathrm{b}}$, D. Maquin ${ }^{\mathrm{a}, \mathrm{b}}$, J. Ragot $^{\mathrm{a}, \mathrm{b}}$ \\ ${ }^{a}$ Université de Lorraine, CRAN, UMR 7039, 2 avenue de la Forêt de Haye Vandoeuvre-les-Nancy, Cedex \\ 54516, France. \\ ${ }^{b}$ CNRS, CRAN, UMR 7039, France.
}

\begin{abstract}
A systematic approach to joint state and time-varying parameter estimation for nonlinear systems is proposed in this paper. Applying the sector nonlinearity transformation to both the system nonlinearities and the time-varying parameters, the original system is equivalently rewritten as a Takagi-Sugeno system with unmeasurable premise variables. A joint state and parameter observer whose parameters are designed by solving an LMI optimization problem is then proposed. The target application is a realistic model of an activated sludge wastewater treatment plant, being an uncertain nonlinear system affected by a time-varying parameter.
\end{abstract}

Keywords: Time-varying nonlinear system, Takagi-Sugeno model, joint state and parameter observer, waste water treatment plant.

\section{INTRODUCTION}

Since most of the control law and fault detection residual design [3], [6] are based on estimated state variables, the observer design for nonlinear systems can be viewed as the heart of system control and model-based diagnosis. Unfortunately, the introduction of time-varying parameters in the system models, needed to accurately represent the system behaviour, leads to more challenging problems in estimation. In this case, conventional observers, essentially developped for time invariant systems cannot be directly used, and so-called adaptive observers developed for joint state and unknown parameter estimation are needed [21]. The main difficulty in estimating the state of such systems comes from the lack of knowledge on the parameter evolution. In the present work, the authors focus on the nonlinear time-varying parameter systems where the parameters are inaccessible (non measurable) and may be considered as model disturbances, uncertainties or faults acting on the system evolution.

Some results have been published on the time-varying systems problem. For example,

Email addresses: souad.bezzaoucha@univ-lorraine.fr (S. Bezzaoucha), benoit.marx@univ-lorraine.fr (B. Marx), didier.maquin@univ-lorraine. fr (D. Maquin), jose.ragoteuniv-lorraine.fr(J. Ragot) 
the state estimation of linear systems with unknown constant or time-varying parameter is respectively addressed in [21] and [11]. Extensions to nonlinear systems are proposed in [1], [4], [16] and [22], but in those works, the parameter is assumed to be constant. Only [9] considers time-varing parameter.

Numerous approaches were proposed in order to deal with nonlinear system estimation or diagnosis [1], [5]. An efficient way consists in rewriting the original nonlinear system in a simplier form, like the Takagi-Sugeno (T-S) model. Originaly introduced by [18], the T-S representation allows to exactly describe nonlinear systems, under the condition that the nonlinearities are bounded. This is reasonable since state variables as well as parameters of physical systems are bounded ([15], [19] and the references therein). The T-S model is a time-varying convex interpolation between linear submodels:

$$
\left\{\begin{aligned}
\dot{x}(t) & =\sum_{i=1}^{r} \mu_{i}(\xi(t))\left(A_{i} x(t)+B_{i} u(t)\right) \\
y(t) & =\sum_{i=1}^{r} \mu_{i}(\xi(t))\left(C_{i} x(t)+D_{i} u(t)\right)
\end{aligned}\right.
$$

where $x(t) \in \mathbb{R}^{n_{x}}$ is the system state, $u(t) \in \mathbb{R}^{n_{u}}$ is the control input and $y(t) \in \mathbb{R}^{m}$ is the system output. $\xi(t) \in \mathbb{R}^{q}$ is the decision variable assumed to be either measurable (as the system output), known (as the system input) or unmeasured (as the system state). The weighting functions $\mu_{i}(\xi(t))$ satisfy the convex sum property:

$$
\left\{\begin{array}{l}
\sum_{i=1}^{r} \mu_{i}(\xi(t))=1 \\
0 \leq \mu_{i}(\xi(t)) \leq 1, \quad i=1, \ldots, r
\end{array}\right.
$$

The representation (1), along with the property (2), allows to extend to nonlinear systems the use of some tools developed in the linear framework, for the stability study, the controller design, the observer synthesis and the diagnosis [15], [19]. A systematic and exact transformation of a nonlinear system into a T-S form, without any loss of informations, is known as the Sector Nonlinearity Transformation (SNT) [19], [14]. Even if SNT leads to T-S models with unmeasurable premise variables, most of the works on T-S systems are devoted to models with known premise variables, since the estimation or diagnosis is obviously easier when the premise variables are accessible. In the following, T-S systems with unmeasurable premise variables (UPM) are studied since they naturaly appear when applying the SNT.

In the present paper, a systematic procedure is presented to deal with the state and parameter estimation for nonlinear time-varying systems. It consists in transforming the original system into a T-S system with unmeasurable premise variables using the SNT. Then a joint state and parameter observer is designed for the T-S system with unknown premise variables. Up to the author's knowledge, this is the first contribution where the joint parameter and state estimation problem is addressed is such a way for the nonlinear systems. Moreover, most of the works devoted to joint parameter and state estimation for nonlinear systems only consider constant parameters, whereas time-varying parameters are here studied. The main result is to establish the convergence conditions of the joint state and parameter estimation errors. The observer gains 
will be derived by solving an LMI optimization problem obtained from the Lyapunov theory. The minimized criterion is the $\mathcal{L}_{2}$-gain of the transfer from the exogeneous inputs to the state and parameter estimation errors. Using the obtained theoretical results, the joint estimation is performed for a wastewater treatment process (WWTP) modeled by an Activated Sludge Model (ASM1 model) [20]. The data measures used for process simulation are those of the European Program Benchmark Cost 624 [2]. Indeed, on a practical point of view, it is shown how to estimate the state of the process using the available measurements and how to estimate a parameter which is varying due to external or internal disturbances. The choice of the known inputs, the time-varying parameter (modeling error), the measures and the operating conditions are made by taking into account the specific features of the Bleesbruck treatment station from Luxemburg. The different steps from the process description as a T-S system to the implementation of time-varying parameter and state estimation are clearly detailed.

The paper is organized as follows. Section 2 introduces the T-S representation of the nonlinear time-varying parameter systems. In section 3, the design of a joint state and parameter observer for T-S system with UPM is presented. Simulation results of the application of the proposed approach to an activated sludge reactor model are given in section 4. Conclusions are detailed in section 5.

\section{Polytopic modelling of nonlinear time-varying parameter systems}

The first contribution of this work is to model nonlinear time-varying systems using the T-S or polytopic representation. For that, each time-varying parameter is rewritten under a particular form.

Let us consider the nonlinear time-varying T-S system represented by equation (3) with $n$ time-varying parameters $\theta_{j}(t)$

$$
\left\{\begin{array}{l}
\dot{x}(t)=\sum_{i=1}^{r} \mu_{i}(\xi(t))\left(A_{i}(\theta(t)) x(t)+B_{i}(\theta(t)) u(t)\right) \\
y(t)=C x(t)
\end{array}\right.
$$

with $\theta(t)=\left[\begin{array}{lll}\theta_{1}(t) & \ldots & \theta_{n}(t)\end{array}\right]^{T}$ and

$$
\begin{aligned}
& A_{i}(\theta(t))=\bar{A}_{i}+\sum_{j=1}^{n} \theta_{j}(t) \bar{A}_{i j} \\
& B_{i}(\theta(t))=\bar{B}_{i}+\sum_{j=1}^{n} \theta_{j}(t) \bar{B}_{i j}
\end{aligned}
$$

Remark 1. With no loss of generality, it is supposed that the matrices $A_{i}(\theta(t))$ and $B_{i}(\theta(t))$ depend on the same time-varying parameters $\theta_{j}(t)$. If a given matrix $A_{i}$ (resp. $B_{i}$ ) does not depend on a given $\theta_{j}(t)$, then $\bar{A}_{i j}$ (resp. $\left.\bar{B}_{i j}\right)$ is null in (4).

For example, if the matrices $A_{i}\left(\theta_{a}(t)\right)$ (resp. $B_{i}\left(\theta_{b}(t)\right)$ ) depend on $\theta_{a} \in \mathbb{R}^{n_{a}}$ (resp $\left.\theta_{b} \in \mathbb{R}^{n_{b}}\right)$, they can be defined as in (4) with $\theta(t)=\left[\theta_{a}^{T}(t), \theta_{b}^{T}(t)\right]^{T}, n=n_{a}+n_{b}$, $\bar{A}_{i j}=0$ for $j=n_{a}+1, \ldots, n$ and $\bar{B}_{i j}=0$ for $j=1, \ldots, n_{a}$. 
According to the SNT [19], each parameter $\theta_{j}(t)$ is expressed as a function of its upper and lower bounds, respectively denoted $\theta_{j}^{1}$ and $\theta_{j}^{2}$ such that:

$$
\theta_{j}(t)=\widetilde{\mu}_{j}^{1}\left(\theta_{j}(t)\right) \theta_{j}^{1}+\widetilde{\mu}_{j}^{2}\left(\theta_{j}(t)\right) \theta_{j}^{2}
$$

where $\widetilde{\mu}_{j}^{1}\left(\theta_{j}(t)\right)$ and $\widetilde{\mu}_{j}^{2}\left(\theta_{j}(t)\right)$ are defined by

$$
\begin{aligned}
& \widetilde{\mu}_{j}^{1}\left(\theta_{j}(t)\right)=\frac{\theta_{j}(t)-\theta_{j}^{2}}{\theta_{j}^{1}-\theta_{j}^{2}} \\
& \widetilde{\mu}_{j}^{2}\left(\theta_{j}(t)\right)=\frac{\theta_{j}^{1}-\theta_{j}(t)}{\theta_{j}^{1}-\theta_{j}^{2}}
\end{aligned}
$$

and satisfy the convex sum property:

$$
\left\{\begin{array}{l}
\widetilde{\mu}_{j}^{1}\left(\theta_{j}(t)\right)+\widetilde{\mu}_{j}^{2}\left(\theta_{j}(t)\right)=1, \forall t \\
0 \leq \widetilde{\mu}_{j}^{i}\left(\theta_{j}(t)\right) \leq 1
\end{array}\right.
$$

Replacing (5) in (4), it becomes:

$$
\begin{aligned}
& A_{i}(\theta(t))=\bar{A}_{i}+\sum_{j=1}^{n} \sum_{k=1}^{2} \widetilde{\mu}_{j}^{k}\left(\theta_{j}(t)\right) \theta_{j}^{k} \bar{A}_{i j} \\
& B_{i}(\theta(t))=\bar{B}_{i}+\sum_{j=1}^{n} \sum_{k=1}^{2} \widetilde{\mu}_{j}^{k}\left(\theta_{j}(t)\right) \theta_{j}^{k} \bar{B}_{i j}
\end{aligned}
$$

The time-varying matrices $A_{i}(\theta(t))$ and $B_{i}(\theta(t))$ can now be written as polytopic matrices. Firstly, due to (7), it follows that

$$
\begin{aligned}
& \sum_{j=1}^{n} \theta_{j}(t) \bar{A}_{i j}=\sum_{j=1}^{n}\left[\left(\widetilde{\mu}_{j}^{1}\left(\theta_{j}(t)\right) \theta_{j}^{1}+\widetilde{\mu}_{j}^{2}\left(\theta_{j}(t)\right) \theta_{j}^{2}\right) \bar{A}_{i j}\right] \\
& =\sum_{j=1}^{n}\left[\left[\left(\widetilde{\mu}_{j}^{1}\left(\theta_{j}(t)\right) \theta_{j}^{1}+\widetilde{\mu}_{j}^{2}\left(\theta_{j}(t)\right) \theta_{j}^{2}\right) \bar{A}_{i j}\right]\left[\prod_{k=1, k \neq j}^{n} \sum_{m=1}^{2} \widetilde{\mu}_{k}^{m}\left(\theta_{k}(t)\right)\right]\right]
\end{aligned}
$$

and thus, equations (8) can be written as:

$$
\begin{aligned}
& A_{i}(\theta(t))=\sum_{j=1}^{2^{n}} \widetilde{\mu}_{j}(\theta(t)) \mathcal{A}_{i j} \\
& B_{i}(\theta(t))=\sum_{j=1}^{2^{n}} \widetilde{\mu}_{j}(\theta(t)) \mathcal{B}_{i j}
\end{aligned}
$$

with

$$
\left\{\begin{aligned}
\widetilde{\mu}_{j}(\theta(t)) & =\prod_{k=1}^{n} \tilde{\mu}_{k}^{\sigma_{j}^{k}}\left(\theta_{k}(t)\right) \\
\mathcal{A}_{i j} & =\bar{A}_{i}+\sum_{k=1}^{n} \theta_{k}^{\sigma_{j}^{k}} \bar{A}_{i k} \\
\mathcal{B}_{i j} & =\bar{B}_{i}+\sum_{k=1}^{n} \theta_{k}^{\sigma_{j}^{k}} \bar{B}_{i k}
\end{aligned}\right.
$$


Then, for $n$ parameters, $2^{n}$ submodels are obtained. It is important to note that an analytical expression of the time-varying matrices $A_{i}(\theta(t))$ and $B_{i}(\theta(t))$ is obtained with convex weighting functions $\widetilde{\mu}_{j}(\theta(t))$ satisfying $\sum_{i=1}^{2^{n}} \widetilde{\mu}_{j}(\theta(t))=1$, where the indices $\sigma_{j}^{k}\left(j=1, \ldots, 2^{n}, k=1, \ldots, n\right)$, equal to 1 or 2 , indicate which partition of the $k^{\text {th }}$ parameter $\left(\widetilde{\mu}_{k}^{1}\right.$ or $\left.\widetilde{\mu}_{k}^{2}\right)$ is involved in the $j^{\text {th }}$ submodel.

The relation between $j$ and the $\sigma_{j}^{k}$ is given by the following equation:

$$
j=2^{n-1} \sigma_{j}^{1}+2^{n-2} \sigma_{j}^{2}+\ldots+2^{0} \sigma_{j}^{n}-\left(2^{1}+2^{2}+\ldots+2^{n-1}\right)
$$

Finally, using equations (10), the nonlinear time-varying T-S system (3) becomes:

$$
\left\{\begin{array}{l}
\dot{x}(t)=\sum_{i=1}^{r} \sum_{j=1}^{2^{n}} \mu_{i}(\xi(t)) \widetilde{\mu}_{j}(\theta(t))\left(\mathcal{A}_{i j} x(t)+\mathcal{B}_{i j} u(t)\right) \\
y(t)=C x(t)
\end{array}\right.
$$

\section{State and time-varying parameter observer}

Considering the obtained T-S model (13), with the weighting functions $\mu_{i}$ and $\widetilde{\mu}_{j}$ depending on the system state $x(t)$ and on the parameter $\theta(t)$, a joint state and parameter observer may be designed and implemented. An $\mathcal{L}_{2}$ attenuation approach is proposed to minimize the effect of the time-varying parameters on the state and parameter error estimations.

The state and parameter observer of system (13) is taken as the following

$$
\left\{\begin{array}{l}
\dot{\hat{x}}(t)=\sum_{i=1}^{r} \sum_{j=1}^{2^{n}} \mu_{i}(\hat{x}(t)) \widetilde{\mu}_{j}(\hat{\theta}(t))\left(\mathcal{A}_{i j} \hat{x}(t)+\mathcal{B}_{i j} u(t)+L_{i j}(y(t)-\hat{y}(t))\right) \\
\dot{\hat{\theta}}(t)=\sum_{i=1}^{r} \sum_{j=1}^{2^{n}} \mu_{i}(\hat{x}(t)) \widetilde{\mu}_{j}(\hat{\theta}(t))\left(-\alpha_{i j} \hat{\theta}(t)+K_{i j}(y(t)-\hat{y}(t))\right) \\
\hat{y}(t)=C \hat{x}(t)
\end{array}\right.
$$

where $L_{i j} \in \mathbb{R}^{n_{x} \times m}, K_{i j} \in \mathbb{R}^{n \times m}$ and $\alpha_{i j} \in \mathbb{R}^{n \times n}$ are the gains to be determined such that the estimated state and parameter converge to the actual system state and parameter.

Let us define the state estimation error $e_{x}(t)$ as

$$
e_{x}(t)=x(t)-\hat{x}(t)
$$

Its dynamics cannot be easily computed directly from the derivative of (15) since in the considered case, the weighting functions depend on the unmeasurable variables $\theta(t)$ and $x(t)$ whereas those of the observer (14) depend on their estimates $\hat{\theta}(t)$ and $\hat{x}(t)$. In order to overcome this difficulty, taking benefits of (2), the state derivative (13) is 
rewritten as follows [8]

$$
\begin{aligned}
\dot{x}(t)=\sum_{i=1}^{r} \sum_{j=1}^{2^{n}}\left[\mu_{i}(\hat{x}(t)) \widetilde{\mu}_{j}(\hat{\theta}(t))\left(\mathcal{A}_{i j} x(t)+\mathcal{B}_{i j} u(t)\right)+\right. \\
\left.\left(\mu_{i}(x(t)) \widetilde{\mu}_{j}(\theta(t))-\mu_{i}(\hat{x}(t)) \widetilde{\mu}_{j}(\hat{\theta}(t))\right)\left(\mathcal{A}_{i j} x(t)+\mathcal{B}_{i j} u(t)\right)\right]
\end{aligned}
$$

This form allows a better comparison of $x(t)$ with $\hat{x}(t)$, since $\mu_{i}(\hat{x}(t)) \widetilde{\mu}_{j}(\hat{\theta}(t))$ not only appears in (14), but also in (16). Let us define:

$$
\begin{aligned}
\Delta A(t) & =\sum_{i=1}^{r} \sum_{j=1}^{2^{n}}\left[\mu_{i}(x(t)) \widetilde{\mu}_{j}(\theta(t))-\mu_{i}(\hat{x}(t)) \widetilde{\mu}_{j}(\hat{\theta}(t))\right] \mathcal{A}_{i j} \\
& =\mathcal{A} \Sigma_{A}(t) E_{A} \\
\Delta B(t) & =\sum_{i=1}^{r} \sum_{j=1}^{2^{n}}\left[\mu_{i}(x(t)) \widetilde{\mu}_{j}(\theta(t))-\mu_{i}(\hat{x}(t)) \widetilde{\mu}_{j}(\hat{\theta}(t))\right] \mathcal{B}_{i j} \\
& =\mathcal{B} \Sigma_{B}(t) E_{B}
\end{aligned}
$$

with

$$
\begin{aligned}
& \mathcal{A}=\left[\begin{array}{lll}
\mathcal{A}_{11} & \ldots & \mathcal{A}_{r 2^{n}}
\end{array}\right], \Sigma_{A}(t)=\operatorname{diag}\left(\delta_{11}(t) I_{n_{x}}, \ldots, \delta_{r 2^{n}}(t) I_{n_{x}}\right), \\
& \mathcal{B}=\left[\begin{array}{lll}
\mathcal{B}_{11} & \ldots & \mathcal{B}_{r 2^{n}}
\end{array}\right], \Sigma_{B}(t)=\operatorname{diag}\left(\delta_{11}(t) I_{n_{u}}, \ldots, \delta_{r 2^{n}}(t) I_{n_{u}}\right), \\
& E_{A}=\left[\begin{array}{lll}
I_{n_{x}} & \ldots & I_{n_{x}}
\end{array}\right]^{T}, E_{B}=\left[\begin{array}{lll}
I_{n_{u}} & \ldots & I_{n_{u}}
\end{array}\right]^{T} \\
& \delta_{i j}(t)=\mu_{i}(x(t)) \tilde{\mu}_{j}(\theta(t))-\mu_{i}(\hat{x}(t)) \widetilde{\mu}_{j}(\hat{\theta}(t))
\end{aligned}
$$

where $\operatorname{diag}\left(A_{1}, \ldots, A_{n}\right)$ denotes a block diagonal matrix with the square matrices $A_{1}, \ldots, A_{n}$ on its diagonal.

Since $\mu_{i}(x(t))$ and $\widetilde{\mu}_{j}(\theta(t))$ satisfy (2) and from the definitions (19), the matrices $\Sigma_{A}(t)$ and $\Sigma_{B}(t)$ are bounded:

$$
\Sigma_{A}^{T}(t) \Sigma_{A}(t) \leq I, \quad \Sigma_{B}^{T}(t) \Sigma_{B}(t) \leq I
$$

Using (17) and (18), the system (16) is then written as an uncertain T-S system given by:

$$
\dot{x}(t)=\sum_{i=1}^{r} \sum_{j=1}^{2^{n}} \mu_{i}(\hat{x}(t)) \widetilde{\mu}_{j}(\hat{\theta}(t))\left(\left(\mathcal{A}_{i j}+\Delta A(t)\right) x(t)+\left(\mathcal{B}_{i j}+\Delta B(t)\right) u(t)\right)
$$

Let us define the parameter estimation error $e_{\theta}(t)$ by

$$
e_{\theta}(t)=\theta(t)-\hat{\theta}(t)
$$

From (14), (21), (15) and (22) the dynamics of the state and parameter estimation errors are given by

$$
\dot{e}_{a}(t)=\sum_{i=1}^{r} \sum_{j=1}^{2^{n}} \mu_{i}(\hat{x}(t)) \widetilde{\mu}_{j}(\hat{\theta}(t))\left(\Phi_{i j} e_{a}(t)+\Psi_{i j}(t) \omega(t)\right)
$$


with

$$
e_{a}(t)=\left(\begin{array}{c}
e_{x}(t) \\
e_{\theta}(t)
\end{array}\right), \omega(t)=\left(\begin{array}{c}
x(t) \\
\theta(t) \\
\dot{\theta}(t) \\
u(t)
\end{array}\right)
$$

and

$$
\begin{aligned}
& \Phi_{i j}=\left(\begin{array}{cc}
\mathcal{A}_{i j}-L_{i j} C & 0 \\
-K_{i j} C & -\alpha_{i j}
\end{array}\right) \\
& \Psi_{i j}(t)=\left(\begin{array}{cccc}
\Delta A(t) & 0 & 0 & \Delta B(t) \\
0 & \alpha_{i j} & I & 0
\end{array}\right)
\end{aligned}
$$

Considering (23), the objective is to design a joint state and parameter observer minimizing the $\mathcal{L}_{2}$ gain of the transfer from $\omega(t)$ to $e_{a}(t)$. The computation of the observer gains $K_{i j}, L_{i j}$ and $\alpha_{i j}$ is detailed in the next theorem.

Theorem 1. There exists a joint robust state and parameter observer (14) for a nonlinear time-varying parameter system (3) with an $\mathcal{L}_{2}$ gain from $\omega(t)$ to $e_{a}(t)$ bounded by $\beta$ $(\beta>0)$ if there exists symmetric positive definite matrices $P_{0} \in \mathbb{R}^{n_{x} \times n_{x}}, P_{1} \in \mathbb{R}^{n \times n}$, matrices $\Gamma_{2}^{0} \in \mathbb{R}^{n_{x} \times n_{x}}, \Gamma_{2}^{1} \in \mathbb{R}^{n \times n}, \Gamma_{2}^{2} \in \mathbb{R}^{n \times n}, \Gamma_{2}^{3} \in \mathbb{R}^{n_{u} \times n_{u}}$ and positive scalars $\beta, \lambda_{1}, \lambda_{2}>0$ solutions of the optimization problem (26)

$$
\min _{P_{0}, P_{1}, R_{i j}, F_{i j}, \bar{\alpha}_{i j}, \lambda_{1}, \lambda_{2}, \Gamma_{2}^{k}} \beta
$$

under the following constraints:

$$
\Gamma_{2}^{k}<\beta I \text { for } k=0,1,2,3
$$

$$
\left(\begin{array}{cccccccc}
Q_{i j}^{11} & -C^{T} F_{i j}^{T} & 0 & 0 & 0 & 0 & P_{0} \mathcal{A} & P_{0} \mathcal{B} \\
* & Q_{i j}^{22} & 0 & \bar{\alpha}_{i j} & P_{1} & 0 & 0 & 0 \\
* & * & Q_{i j}^{33} & 0 & 0 & 0 & 0 & 0 \\
* & * & * & -\Gamma_{2}^{1} & 0 & 0 & 0 & 0 \\
* & * & * & * & -\Gamma_{2}^{2} & 0 & 0 & 0 \\
* & * & * & * & * & Q_{i j}^{66} & 0 & 0 \\
* & * & * & * & * & * & -\lambda_{1} I & 0 \\
* & * & * & * & * & * & 0 & -\lambda_{2} I
\end{array}\right)<0
$$

for $i=1, \ldots, r$ and $j=1,2^{n}$, with

$$
\begin{aligned}
& Q_{i j}^{11}=P_{0} \mathcal{A}_{i j}+\mathcal{A}_{i j}^{T} P_{0}-R_{i j} C-C^{T} R_{i j}^{T}+I_{n_{x}} \\
& Q_{i j}^{22}=-\bar{\alpha}_{i j}-\bar{\alpha}_{i j}^{T}+I_{n} \\
& Q_{i j}^{33}=-\Gamma_{2}^{0}+\lambda_{1} E_{A}^{T} E_{A} \\
& Q_{i j}^{66}=-\Gamma_{2}^{3}+\lambda_{2} E_{B}^{T} E_{B}
\end{aligned}
$$

The observer gains are given by

$$
\left\{\begin{array}{l}
L_{i j}=P_{0}^{-1} R_{i j} \\
K_{i j}=P_{1}^{-1} F_{i j} \\
\alpha_{i j}=P_{1}^{-1} \bar{\alpha}_{i j}
\end{array}\right.
$$

Proof 1. The proof of theorem 1 can be found in appendix A. 


\section{Application to a wastewater treatment plant}

\subsection{Process description}

The considered wastewater treatment plant is issued from [20] and the considered T-S model without taking into account the time-varying parameter [13].

The activated sludge wastewater treatment consists in mixing wastewater with a bacteria mixture in order to degrade the pollutants contained in the water. The polluted water circulates in an aeration basin in which the bacterial biomass degrades the polluted matter. Micro-organisms gather together in colonial structures called flocs and produce sludges. The mixed liquor is then sent to a clarifier where the separation of the purified water and the flocs is made by gravity. A fraction of the settled sludges is recycled towards the reactor to maintain its capacity of purification. The purified water is thrown back in the natural environment.

Only a part of the European Program Cost 624 Benchmark is considered. Usually, a configuration with a single tank and a settler/clarifier is used. However, the data used for simulation are generated with the complete ASM1 model $\left(n_{x}=13\right)$ [7], in order to represent a realistic behavior of a WWTP. In order to ease the T-S modelling, the observer design is based on a reduced model $\left(n_{x}=6\right)$ [20]. The simplified model involves the following six components: the chemical oxygen demand (DCO) $X_{D C O}$, oxygen $S_{O}$, heterotrophic biomass $X_{B H}$, ammonia $S_{N H}$, nitrate $S_{N O}$ and autotrophic biomass $X_{B A}$. Thus, the state vector is defined as:

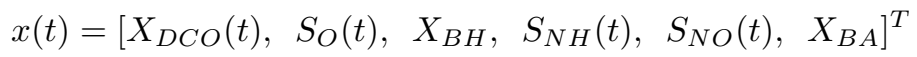

In conformity with the benchmark of the European Program Cost 624 [2] and with the real operating condition of the wastewater treatment plant (Bleesbruck from Luxemburg), the considered output and input vectors are:

$$
\begin{aligned}
& y(t)=\left[X_{D C O}(t), \quad S_{O}(t), \quad S_{N H}(t), \quad S_{N O}(t)\right]^{T} \\
& u(t)=\left[\begin{array}{lll}
X_{D C O, i n}(t), & q_{a}(t), & q_{i n}(t)
\end{array}\right]^{T}
\end{aligned}
$$

The variables $q_{i n}(t)$ and $q_{a}(t)$ respectively represent the flowrate input and the air flow of the bioreactor.

The dynamic equations describing the system are the following

$$
\left\{\begin{aligned}
\dot{X}_{D C O}(t)= & -\frac{1}{Y_{h}}\left[\varphi_{1}(t)+\varphi_{2}(t)\right]+\left(1-f_{p}\right)\left(\varphi_{4}(t)+\varphi_{5}(t)\right)+D_{1}(t) \\
\dot{S}_{O}(t)= & \frac{Y_{h}-1}{Y_{h}} \varphi_{1}(t)+\frac{Y_{a}-4.57}{Y_{a}} \varphi_{3}(t)+D_{2}(t) \\
\dot{S}_{N H}(t)= & -i_{x b}\left[\varphi_{1}(t)+\varphi_{2}(t)\right]-\left[i_{x b}+\frac{1}{Y_{a}}\right] \varphi_{3}(t) \\
& +\left(i_{x b}-f_{p} i_{x p}\right)\left[\varphi_{4}(t)+\varphi_{5}(t)\right]+D_{3}(t) \\
\dot{S}_{N O}(t)= & \frac{Y_{h}-1}{2.86 Y_{h}} \varphi_{2}(t)+\frac{1}{Y_{a}} \varphi_{3}(t)+D_{4}(t) \\
\dot{X}_{B H}(t)= & \varphi_{1}(t)+\varphi_{2}(t)-\varphi_{4}(t)+D_{5}(t) \\
\dot{X}_{B A}(t)= & \varphi_{3}(t)-\varphi_{5}(t)+D_{6}(t)
\end{aligned}\right.
$$


where $Y_{a}, \quad Y_{h}, \quad f_{p}, \quad i_{x b}, \quad i_{x p}$ are constant coefficients and $\varphi_{1}(t), \cdots, \varphi_{5}(t)$ are given by:

$$
\left\{\begin{array}{l}
\varphi_{1}(t)=\mu_{h} \frac{X_{D C O}(t)}{K_{D C O}+X_{D C O}(t)} \frac{S_{O}(t)}{K_{O H}+S_{O}(t)} X_{B H}(t) \\
\varphi_{2}(t)=\mu_{h} \eta_{N O g} \frac{X_{D C O}(t)}{K_{D C O}+X_{D C O}(t)} \frac{S_{N O}(t)}{K_{N O}+S_{N O}(t)} \frac{K_{O H}}{K_{O H}+S_{O}(t)} X_{B H}(t) \\
\varphi_{3}(t)=\mu_{a} \frac{S_{N H}(t)}{K_{N H, A}+S_{N H}(t)} \frac{S_{O}(t)}{K_{O, A}+S_{O}(t)} X_{B A}(t) \\
\varphi_{4}(t)=b_{H} X_{B H}(t) \\
\varphi_{5}(t)=b_{A} X_{B A}(t)
\end{array}\right.
$$

According to the benchmark [2], it is supposed that the dissolved oxygen concentration at the reactor input $\left(S_{O, \text { in }}\right)$ is null, that $S_{N O, \text { in }} \cong 0$ and $X_{B A, i n} \cong 0$. The input/output balance is defined by:

$$
\begin{aligned}
& D_{1}(t)=D_{i n}(t)\left[X_{D C O, i n}(t)-X_{D C O}(t)\right] \\
& D_{2}(t)=D_{i n}(t)\left[-S_{O}(t)\right]+K q_{a}(t)\left[S_{O, s a t}-S_{O}(t)\right] \\
& D_{3}(t)=D_{i n}(t)\left[S_{N H, i n}(t)-S_{N H}(t)\right] \\
& D_{4}(t)=D_{i n}(t)\left[-S_{N O}(t)\right] \\
& D_{5}(t)=D_{i n}(t)\left[X_{B H, i n}(t)-X_{B H}(t)+\frac{f_{r}\left(1-f_{w}\right)}{f_{r}+f_{w}} X_{B H}(t)\right] \\
& D_{6}(t)=D_{i n}(t)\left[-X_{B A}(t)+\frac{f_{r}\left(1-f_{w}\right)}{f_{r}+f_{w}} X_{B A}(t)\right] \\
& D_{5}(t)=D_{i n}(t)\left[X_{B H, i n}(t)-\frac{f_{w}\left(1+f_{r}\right)}{f_{r}+f_{w}} X_{B H}(t)\right] \\
& D_{6}(t)=D_{i n}(t)\left[-X_{B A}(t)-\frac{f_{w}\left(1+f_{r}\right)}{f_{r}+f_{w}} X_{B A}(t)\right]
\end{aligned}
$$

with $D_{i n}(t)=\frac{q_{i n}(t)}{V}$

\subsection{Polytopic $T-S$ representation}

Since the transformation of the nonlinear system (34) into a T-S model does not constitute the main contribution, and for lack of space, only the essential points are given in the following. For further details on this procedure, the reader may refer to [13], [12].

Considering the process (34), it is natural to define the following premise variables involved in the system nonlinearities:

$$
\begin{aligned}
& \xi_{1}(t)=\frac{q_{i n}(t)}{V} \\
& \xi_{2}(t)=\frac{X_{D C O}(t)}{K_{D C O}+X_{D C O}(t)} \frac{S_{O}(t)}{K_{O H}+S_{O}(t)} \\
& \xi_{3}(t)=\frac{X_{D C O}(t)}{K_{D C O}+X_{D C O}(t)} \frac{S_{N O}(t)}{K_{N O}+S_{N O}(t)} \frac{K_{O H}}{K_{O H}+S_{O}(t)} \\
& \xi_{4}(t)=\frac{1}{K_{O A}+S_{O}(t)} \frac{S_{N H}(t)}{K_{N H, A}+S_{N H}(t)} X_{B A}(t)
\end{aligned}
$$


Then system (34) can be written in a quasi-LPV form

$$
\dot{x}(t)=A(\xi) x(t)+B(\xi) u(t)
$$

with matrices $A(\xi)$ and $B(\xi)$ depending on the premise variables previously defined:

$$
\begin{aligned}
& A(\xi)=\left[\begin{array}{cccccc}
a_{1,1}\left(\xi_{1}\right) & 0 & 0 & 0 & a_{1,5}\left(\xi_{2}, \xi_{3}\right) & a_{1,6} \\
0 & a_{2,2}\left(\xi_{1}, \xi_{4}\right) & 0 & 0 & a_{2,5}\left(\xi_{2}\right) & 0 \\
0 & a_{3,2}\left(\xi_{4}\right) & a_{3,3}\left(\xi_{1}\right) & 0 & a_{3,5}\left(\xi_{2}, \xi_{3}\right) & a_{3,6} \\
0 & a_{4,2}\left(\xi_{4}\right) & 0 & a_{4,4}\left(\xi_{1}\right) & a_{4,5}\left(\xi_{3}\right) & 0 \\
0 & 0 & 0 & 0 & a_{5,5}\left(\xi_{1}, \xi_{2}, \xi_{3}\right) & 0 \\
0 & a_{6,2}\left(\xi_{4}\right) & 0 & 0 & 0 & a_{6,6}\left(\xi_{1}\right)
\end{array}\right] \\
& B(\xi)=\left[\begin{array}{cc}
b_{1,1}\left(\xi_{1}\right) & 0 \\
0 & K S_{O, s a t} \\
0 & 0 \\
0 & 0 \\
0 & 0 \\
0 & 0
\end{array}\right]
\end{aligned}
$$

where $a_{1,1}\left(\xi_{1}\right)=a_{3,3}\left(\xi_{1}\right)=a_{4,4}\left(\xi_{1}\right)=-b_{1,1}\left(\xi_{1}\right)=-\xi_{1}(t)$ and:

$$
\begin{array}{ll}
a_{1,5}\left(\xi_{2}, \xi_{3}\right) & =-\frac{\mu_{h}}{Y_{h}} \xi_{2}(t)+\left(1-f_{p}\right) b_{H}-\frac{\mu_{h} \eta_{N O g}}{Y_{h}} \xi_{3}(t) \\
a_{1,6} & =\left(1-f_{p}\right) b_{a} \\
a_{2,2}\left(\xi_{1}, \xi_{4}\right) & =-\xi_{1}(t)-K q_{a}-\frac{4.57-Y_{a}}{Y_{a}} \mu_{a} \xi_{4}(t) \\
a_{2,5}\left(\xi_{2}\right) & =\frac{Y_{h}-1}{Y_{h}} \mu_{h} \xi_{2}(t) \\
a_{3,2}\left(\xi_{4}\right) & =-\left(i_{x b}+\frac{1}{Y_{a}}\right) \mu_{a} \xi_{4}(t) \\
a_{3,5}\left(\xi_{2}, \xi_{3}\right) & =\left(i_{x b}-f_{p} i_{x p}\right) b_{H}-i_{x b} \mu_{h} \xi_{2}(t)-i_{x b} \mu_{h} \eta_{N O g} \xi_{3}(t) \\
a_{3,6} & =\left(i_{x b}-f_{p} i_{x p}\right) b_{A} \\
a_{4,2}\left(\xi_{4}\right) & =\frac{1}{Y_{a}} \mu_{A} \xi_{4}(t) \\
a_{4,5}\left(\xi_{3}\right) & =\frac{Y_{h}-1}{2.86 Y_{h}} \mu_{h} \eta_{N O g} \xi_{3}(t) \\
a_{5,5}\left(\xi_{1}, \xi_{2}, \xi_{3}\right) & =\mu_{h} \xi_{2}(t)-b_{H}+\xi_{1}(t)\left[\frac{f_{w}\left(1+f_{r}\right)}{f_{r}+f_{w}}-1\right]+\mu_{h} \eta_{N O g} \xi_{3}(t) \\
a_{6,2}\left(\xi_{4}\right) & =\mu_{a} \xi_{4}(t) \\
a_{6,6}\left(\xi_{1}\right) & =\xi_{1}(t)\left[\frac{f_{w}\left(1+f_{r}\right)}{f_{r}+f_{w}}-1\right]-b_{A}
\end{array}
$$

The premise variables $\xi_{j}(t), j=1, \ldots, 4$ are re-written using the convex polytopic transformation:

$$
\xi_{j}(t)=F_{j, 1}\left(\xi_{j}(t)\right) \xi_{j, 1}+F_{j, 2}\left(\xi_{j}(t)\right) \xi_{j, 2}
$$

where the scalars $\xi_{j, 1}, \xi_{j, 2}$ and the functions $F_{j, 1}\left(\xi_{j}\right), F_{j, 2}\left(\xi_{j}\right)$ are defined by

$$
\begin{aligned}
\xi_{j, 1} & =\max _{x, u}\left\{\xi_{j}(t)\right\} \\
\xi_{j, 2} & =\min _{x, u}\left\{\xi_{j}(t)\right\} \\
F_{j, 1}\left(\xi_{j}(t)\right) & =\frac{\xi_{j}(t)-\xi_{j, 2}}{\xi_{j, 1}-\xi_{j, 2}} \\
F_{j, 2}\left(\xi_{j}(t)\right) & =\frac{\xi_{j, 1}-\xi_{j}(t)}{\xi_{j, 1}-\xi_{j, 2}}
\end{aligned}
$$


In order to obtain the T-S structure from the quasi-LPV system (38), each variable $\xi_{j}(t)$ is decomposed according to (40) and multiplied by $\prod_{k=1, \neq j}^{4}\left(F_{j, 1}+F_{j, 2}\right)=1$.

Similarly to the procedure described in section 2 , the obtained weighting functions are defined by:

$$
\mu_{i}(\xi(t))=F_{1, \sigma_{i}^{1}}\left(\xi_{1}(t)\right) F_{2, \sigma_{i}^{2}}\left(\xi_{2}(t)\right) F_{3, \sigma_{i}^{3}}\left(\xi_{3}(t)\right) F_{4, \sigma_{i}^{4}}\left(\xi_{4}(t)\right)
$$

The matrices $A_{i}(\theta(t))$ and $B_{i}(\theta(t))$ are obtained by setting $\xi_{j}(t)=\xi_{j, \sigma_{i}^{j}}(t)$ in $A(\xi)$ and $B(\xi)$, resulting in:

$$
\begin{gathered}
A_{i}=\left[\begin{array}{cccccc}
a_{1,1}\left(\xi_{1, \sigma_{i}^{1}}\right) & 0 & 0 & 0 & a_{1,5}\left(\xi_{2, \sigma_{i}^{2}}, \xi_{3, \sigma_{i}^{3}}\right) & a_{1,6} \\
0 & a_{2,2}\left(\xi_{1, \sigma_{i}^{1}}, \xi_{4, \sigma_{i}^{4}}\right) & 0 & 0 & a_{2,5}\left(\xi_{2, \sigma_{i}^{2}}\right) & 0 \\
0 & a_{3,2}\left(\xi_{4, \sigma_{i}^{4}}\right) & a_{3,3}\left(\xi_{1, \sigma_{i}^{1}}\right) & 0 & a_{3,5}\left(\xi_{2, \sigma_{i}^{2}}, \xi_{3, \sigma_{i}^{3}}\right) & a_{3,6} \\
0 & a_{4,2}\left(\xi_{4, \sigma_{i}^{4}}\right) & 0 & a_{4,4}\left(\xi_{1, \sigma_{i}^{1}}\right) & a_{4,5}\left(\xi_{3, \sigma_{i}^{3}}\right) & 0 \\
0 & 0 & 0 & 0 & a_{5,5}\left(\xi_{1, \sigma_{i}^{1}}, \xi_{2, \sigma_{i}^{2}}, \xi_{3, \sigma_{i}^{3}}\right) & 0 \\
0 & a_{6,2}\left(\xi_{4, \sigma_{i}^{4}}\right) & 0 & 0 & 0 & a_{6,6}\left(\xi_{1, \sigma_{i}^{1}}\right)
\end{array}\right] \\
B_{i}=\left[\begin{array}{ccc}
b_{1,1}\left(\xi_{1, \sigma_{i}^{1}}\right) & 0 \\
0 & K S_{O, s a t} \\
0 & 0 \\
0 & 0 \\
0 & 0 \\
0 & 0
\end{array}\right]
\end{gathered}
$$

Thus, the nonlinear model (38) is equivalently written under the T-S form:

$$
\begin{aligned}
\dot{x}(t) & =\sum_{i=1}^{16} \mu_{i}(x, u)\left(A_{i} x(t)+B_{i} u(t)\right) \\
y(t) & =C x(t)
\end{aligned}
$$

with the output matrix defined as:

$$
C=\left(\begin{array}{llllll}
1 & 0 & 0 & 0 & 0 & 0 \\
0 & 1 & 0 & 0 & 0 & 0 \\
0 & 0 & 0 & 1 & 0 & 0 \\
0 & 0 & 0 & 0 & 1 & 0
\end{array}\right)
$$

\subsection{Time-varying parameter in the T-S form of the ASM1 model}

The T-S model (47) is obtained under the hypothesis that the heterotrophic biomass is homogenous and then the mortality coefficient $b_{H}$ is constant and equal to its nominal value $b_{H n}=0.3$.

Due to a change of operating condition, the coefficient $b_{H}$ may vary. In order to take into account this variation, a bounded time-varying parameter $\theta_{1}(t)$ is introduced, and $b_{H}$ is defined by

$$
b_{H}\left(\theta_{1}(t)\right)=b_{H n}+0.2 \theta_{1}(t), \theta_{1}(t) \in[0,1]
$$


This allows to represent a variation up to $66 \%$ of the nominal value. Since $b_{H}\left(\theta_{1}(t)\right)$ appears in $a_{1,5}, a_{3,5}$ and $a_{5,5}$, the time-varying matrices $A_{i}(\theta(t))$ are written as $A_{i}(\theta(t))$ $=\bar{A}_{i}+\theta_{1}(t) \bar{A}_{i 1}$ with

$$
\bar{A}_{i 1}=\left[\begin{array}{cccccc}
0 & 0 & 0 & 0 & 0.2 & 0 \\
0 & 0 & 0 & 0 & 0 & 0 \\
0 & 0 & 0 & 0 & 0.2 & 0 \\
0 & 0 & 0 & 0 & 0 & 0 \\
0 & 0 & 0 & 0 & 0.2 & 0 \\
0 & 0 & 0 & 0 & 0 & 0
\end{array}\right]
$$

$\bar{A}_{i}$ being obtained from $A_{i}(\theta(t))$ considering $\theta_{1}(t)=0$ (nominal case). For the following simulations, $b_{H}(t)$ is taken as depicted in figure 3 .

\subsection{Results and discussions}

The data used for simulation are generated with the complete ASM1 model described with 13 state variables [7], in order to represent a realistic behaviour of a WWTP. Even if the observer design is based on a T-S form of the reduced model $\left(n_{x}=6\right)$ and is fed by the data from the complete ASM1 model, it will be seen that the estimation results are more than satisfactory.

Applying theorem 1, the joint state and parameter observer (14) is designed by computing all the gains $K_{i j}, L_{i j}$ and $\alpha_{i j}$ (the numerical values are not given here due to space limitation) such that the convergence LMI conditions given in theorem 1 hold. The solution of the optimization problem under LMI constraints was obtained with the use of the SeDuMi solver and Matlab software. Matlab was also used for the system simulations.

Remark 2. SeDuMi (for Self-Dual-Minimization) [17] is a free add-on Matlab package for solving Linear Matrix Inequality (LMI) problems. It allows to solve optimization problems with linear, quadratic and semidefiniteness constraints. Some of SeDuMi characteristics [10]:

- Allows the use of complex or real data and variables.

- Works with both equality and inequality constraints.

- Gives data in sparse format, leading to significant speed benifits (reduced memory burden) and low computation time compared to other solvers.

- Displays the solution directly in matrix format and indicators show which inequality constraints are satisfied.

- Free software.

In order to illustrate the time-varying parameter effect on the system trajectory, figure 1 depicts the state variables in the nominal case (with a constant parameter $b_{H}=0.3$ ) and in the considered case (with the parameter variation affecting $b_{H}$ ). Figure 1 clearly shows that the variation of $b_{H}$ has a significant effect on most of system variables. Comparisons between the actual state variable, the unknown time-varying parameter and their respective estimates are depicted in figures 2 and 3. 

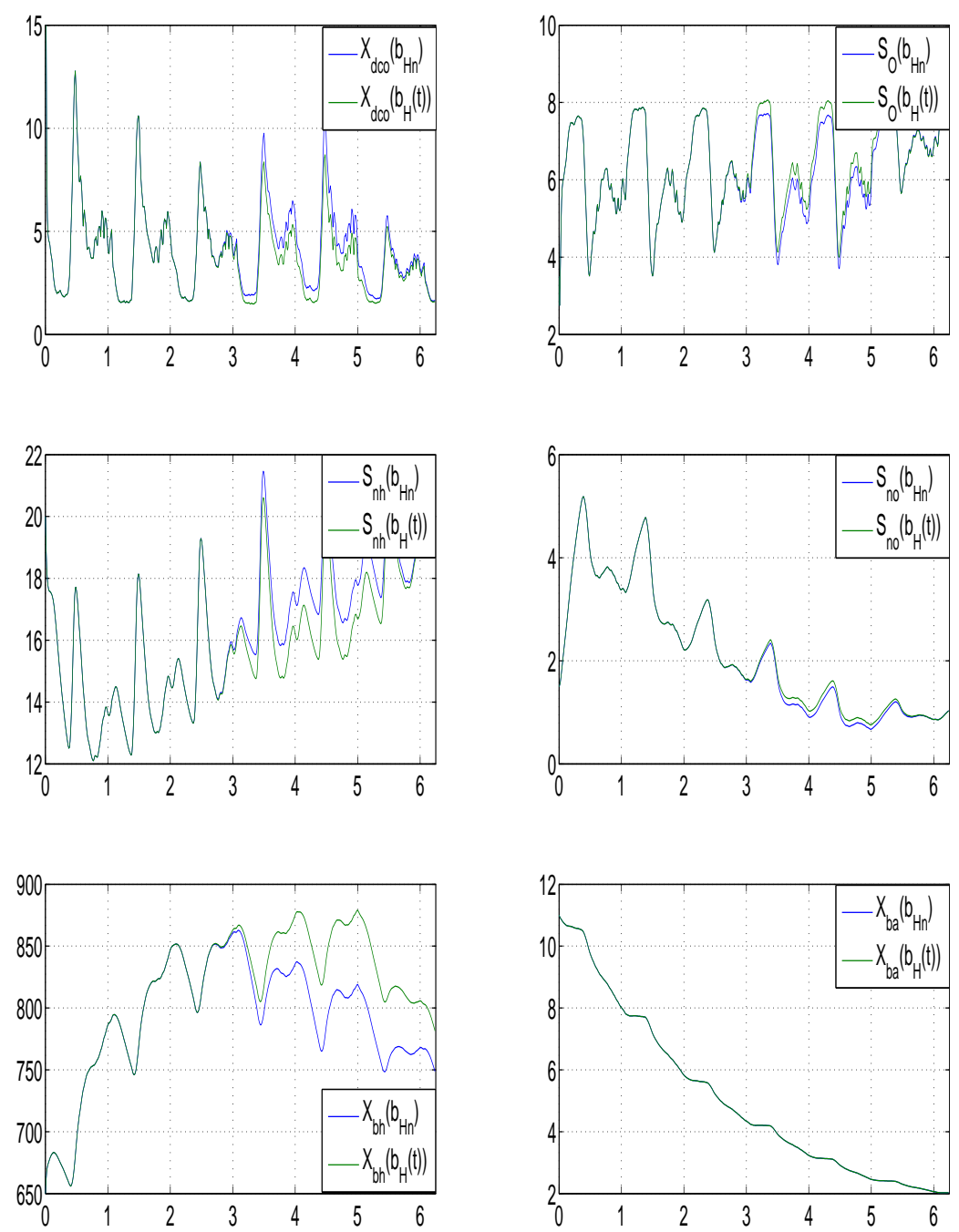

Figure 1: Time-varying parameter effect 

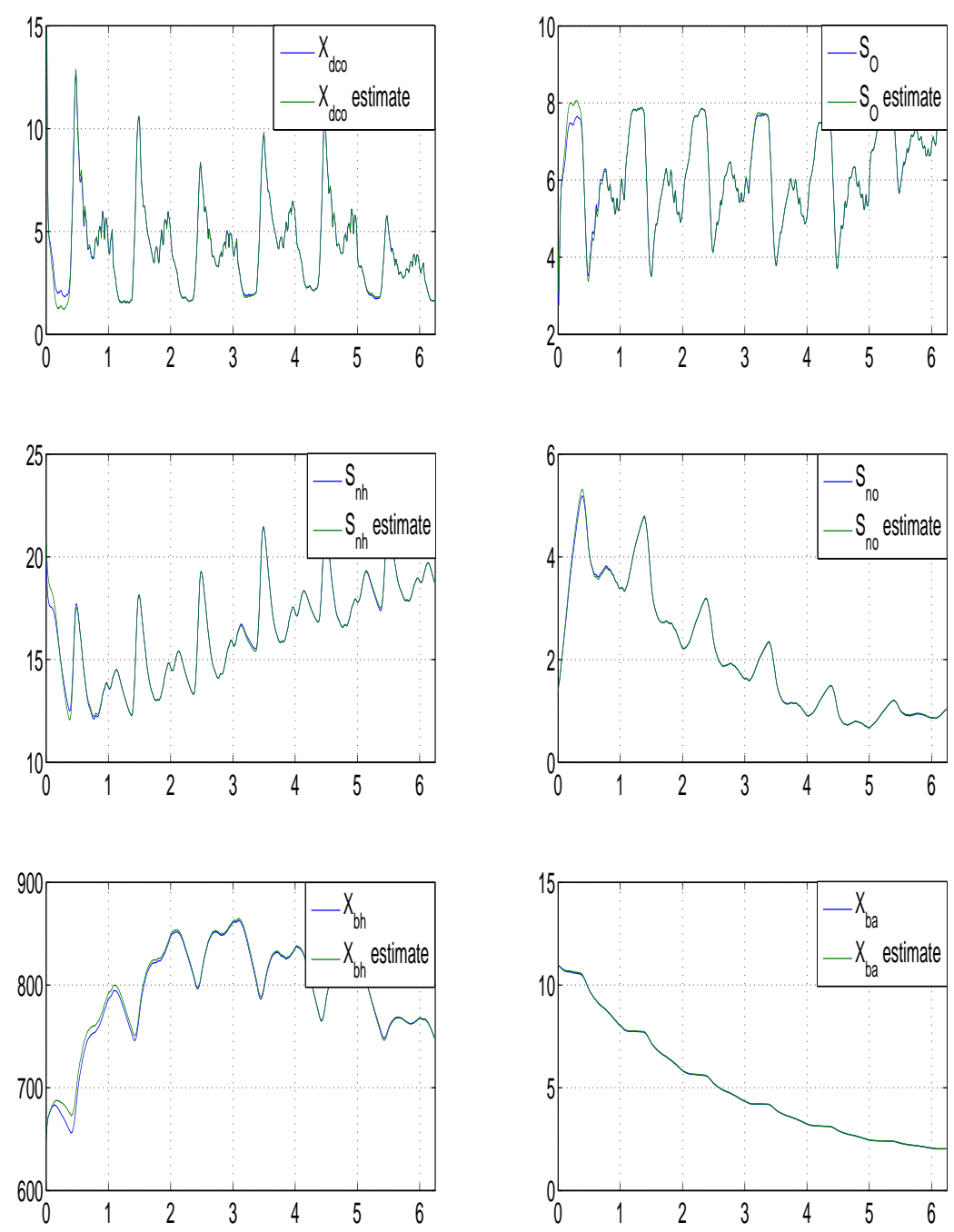

Figure 2: Actual and estimated states 


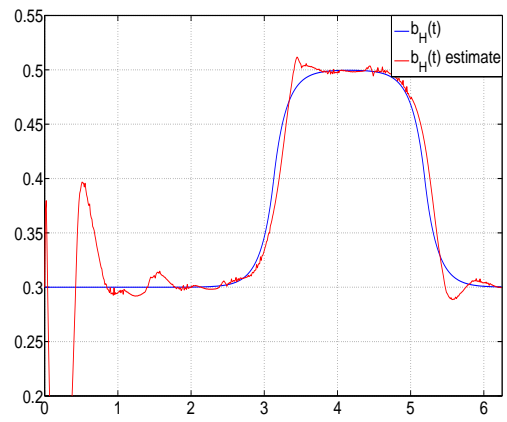

Figure 3: Heterotrophic biomass $b_{H}(t)=b_{H n}+0.2 \theta_{1}(t)$ and its estimate

From the depicted figures, one can conclude on the effectiveness of the synthesized joint state and time-varying parameter observer, since the six states are accurately estimated with an also really good estimation of the time-varying parameter $b_{H}(t)$. One can note that the slight degradation at the start of the simulation of the parameter estimation is due to the initialization error since the system and the observer state are respectively initialized with the following vectors

$$
\begin{aligned}
& x_{0}=\left(\begin{array}{llllll}
30 & 3.5 & 20 & 1.5 & 650 & 11
\end{array}\right)^{T} \\
& \hat{x}_{0}=\left(\begin{array}{llllll}
27 & 4.2 & 21 & 1.4 & 652 & 11
\end{array}\right)^{T}, \hat{\theta}_{0}=0
\end{aligned}
$$

The obtained results show that the state and parameter estimations are good enough with an attenuation level $\beta=2.610^{-2}$.

\section{Conclusion}

In the present paper, a new systematic procedure is presented to deal with the state and parameter estimation for nonlinear time-varying systems. It consists in transforming the original system into a Takagi-Sugeno model based on the sector nonlinearity approach and the convex polytopic transformation. This transformation has the major interest to exactly represent the system without any loss of information. A new parameter and state observer for the time-varying T-S systems with unmeasured premise variables is proposed based on the $\mathcal{L}_{2}$ approach.

The considered procedure is the following: from the nonlinear time-varying equations of the process, a global T-S model of the system is proposed. The proposed observer is then synthetized by solving the LMI optimization problem.

The concrete case of an activated sludge reactor model is studied with modeling errors (function of a time-varying parameter). It was clearly shown that the variation of the heterotrophic biomass $b_{H}$ has a significant effect on most of the system variables. From this observation, one can easily agree with the need for joint parameter and state estimation. 
The obtained results from the application example illustrate clearly the proposed approach performance. Therefore, as a future work, the authors intend to apply this procedure for fault detection and estimation and use the results for a Fault Tolerant Control (FTC) synthesis.

\section{Appendix A. Proof of theorem 1}

In the proof of theorem 1, the following lemma is used:

Lemma Appendix A.1. [23] Consider two matrices $X$ and $Y$ with appropriate dimensions, a time-varying matrix $\Delta(t)$ and a positive scalar $\varepsilon$. The following property is verified

$$
X^{T} \Delta^{T}(t) Y+Y^{T} \Delta(t) X \leq \varepsilon X^{T} X+\varepsilon^{-1} Y^{T} Y
$$

for any $\Delta(t)$ satisfying $\Delta^{T}(t) \Delta(t) \leq I$.

The proof of theorem 1 relies on the following steps.

1) Let us consider the following quadratic Lyapunov function

$$
V\left(e_{a}(t)\right)=e_{a}^{T}(t) P e_{a}(t), P=P^{T}>0
$$

Using (23), its time derivative is given by

$$
\begin{aligned}
& \dot{V}(t)=\sum_{i=1}^{r} \sum_{j=1}^{2^{n}} \mu_{i}(\hat{x}(t)) \widetilde{\mu}_{j}(\hat{\theta}(t))\left[e_{a}^{T}(t)\left(\Phi_{i j}^{T} P+P \Phi_{i j}\right) e_{a}(t)\right. \\
& \left.+e_{a}^{T}(t) P \Psi_{i j}(t) \omega(t)+\omega^{T}(t) \Psi_{i j}^{T}(t) P e_{a}(t)\right]
\end{aligned}
$$

It is known that $e_{a}(t)$ asymptotically converges toward zero when $\omega(t)=0$ and that, when $\omega(t) \neq 0$, the $\mathcal{L}_{2}$ gain from $\omega(t)$ to $e_{a}(t)$ is bounded by $\beta$ if the following inequality holds

$$
\dot{V}(t)+e_{a}^{T}(t) e_{a}(t)-\omega^{T}(t) \Gamma_{2} \omega(t)<0
$$

with

$$
\Gamma_{2}=\operatorname{diag}\left(\Gamma_{2}^{k}\right), \Gamma_{2}^{k}<\beta I, \text { for } k=0,1,2,3
$$

Remark 3. Instead of optimizing the matrix $\Gamma_{2}$ as mentioned in theorem 1, it is also possible to choose $\Gamma_{2}$ in order to attenuate the transfer from some particular components of $\omega(t)$ to $e_{a}(t)$.

2) According to the expression of the derivative of the Lyapunov function, let us rewrite the constraint (A.4). Using (A.3), (A.4) becomes:

$$
\begin{aligned}
& \sum_{i=1}^{r} \sum_{j=1}^{2^{n}} \mu_{i}(\hat{x}(t)) \mu_{j}(\hat{\theta}(t))\left(\begin{array}{c}
e_{a}(t) \\
\omega(t)
\end{array}\right)^{T} \\
& \left(\left(\begin{array}{cc}
\Phi_{i j}^{T} P+P \Phi_{i j}+I_{n_{x}+n} & P \Psi_{i j}(t) \\
\Psi_{i j}^{T}(t) P & -\Gamma_{2}
\end{array}\right)\right)\left(\begin{array}{c}
e_{a}(t) \\
\omega(t)
\end{array}\right)<0
\end{aligned}
$$


The chosen Lyapunov matrix $P$ is block diagonal and defined by:

$$
P=\operatorname{diag}\left(P_{0}, P_{1}\right)
$$

From (22), (25), (A.5) and (A.7), (A.6) holds if

$$
\sum_{i=1}^{r} \sum_{j=1}^{2^{n}} \mu_{i}(\hat{x}(t)) \tilde{\mu}_{j}(\hat{\theta}(t))\left(Q_{i j}+\mathcal{Q}(t)+\mathcal{Q}^{T}(t)\right)<0
$$

with:

$$
\begin{gathered}
Q_{i j}=\left(\begin{array}{cccccc}
\bar{Q}_{i j}^{11} & -C^{T} K_{i j}^{T} P_{1} & 0 & 0 & 0 & 0 \\
* & -P_{1} \alpha_{i j}-\alpha_{i j}^{T} P_{1}+I_{n} & 0 & P_{1} \alpha_{i j} & P_{1} & 0 \\
* & * & -\Gamma_{2}^{0} & 0 & 0 & 0 \\
* & * & * & -\Gamma_{2}^{1} & 0 & 0 \\
* & * & * & * & -\Gamma_{2}^{2} & 0 \\
* & * & * & * & * & -\Gamma_{2}^{3}
\end{array}\right) \\
\bar{Q}_{i j}^{11}=P_{0} \mathcal{A}_{i j}+\mathcal{A}_{i j}^{T} P_{0}-P_{0} L_{i j} C-C^{T} L_{i j}^{T} P_{0}+I_{n_{x}} \\
\mathcal{Q}(t)=\left(\begin{array}{cccccc}
0 & 0 & P_{0} \Delta A(t) & 0 & 0 & P_{0} \Delta B(t) \\
0 & 0 & 0 & 0 & 0 & 0 \\
0 & 0 & 0 & 0 & 0 & 0 \\
0 & 0 & 0 & 0 & 0 & 0 \\
0 & 0 & 0 & 0 & 0 & 0 \\
0 & 0 & 0 & 0 & 0 & 0
\end{array}\right)
\end{gathered}
$$

3) At this stage, if (A.8) holds, it implies (A.4) and then proove the theorem 1. However, (A.8) involves the uncertain term $\mathcal{Q}(t)$ which is time-varying. It is then preferable to bound that term using constants. Based on (17) and (18), the time-varying term (A.11) can be expressed as:

$$
\mathcal{Q}(t)=\left(\begin{array}{c}
P_{0} \mathcal{A} \\
0 \\
0 \\
0 \\
0 \\
0
\end{array}\right) \Sigma_{A}(t)\left(\begin{array}{c}
0 \\
0 \\
E_{A} \\
0 \\
0 \\
0
\end{array}\right)^{T}+\left(\begin{array}{c}
P_{0} \mathcal{B} \\
0 \\
0 \\
0 \\
0 \\
0
\end{array}\right) \Sigma_{B}(t)\left(\begin{array}{c}
0 \\
0 \\
0 \\
0 \\
0 \\
E_{B}
\end{array}\right)^{T}
$$

Using lemma 1 and property (20), there exists positive scalars $\lambda_{1}$ and $\lambda_{2}$, such that

$$
\mathcal{Q}(t)+\mathcal{Q}^{T}(t)<\operatorname{diag}\left(\mathcal{Q}^{1}, 0, \lambda_{1} E_{A}^{T} E_{A}, 0,0, \lambda_{2} E_{B}^{T} E_{B}\right)
$$

with:

$$
\mathcal{Q}^{1}=\lambda_{1}^{-1} P_{0} \mathcal{A} \mathcal{A}^{T} P_{0}+\lambda_{2}^{-1} P_{0} \mathcal{B B}^{T} P_{0}
$$

for $i=1, \ldots, r$ and $j=1, \ldots, 2^{n}$.

From inequality (A.13), using the variable changes (30) and with some Schur complements on the term $\mathcal{Q}^{1}$, it follows that (28) implies

$$
Q_{i j}+\mathcal{Q}(t)+\mathcal{Q}^{T}(t)<0
$$

4) All the elements are now set up for the conclusion. Since $\mu_{i}(\hat{x}(t))$ and $\widetilde{\mu}_{j}(\hat{\theta}(t))$ satisfy (2), (28) also implies (A.8) and then (A.4). As a consequence, the $\mathcal{L}_{2}$-gain of the transfer from $\omega(t)$ to $e_{a}(t)$ is bounded by $\beta$, which achieves the proof. 


\section{References}

[1] Alcorta Garcia, E. and Frank, P. M. [1997], 'Deterministic nonlinear observerbased approaches to fault diagnosis: a survey', Control Engineering Practice 5(5), 663-670.

[2] Alex, J., Béteau, J. F., Copp, J. B., Hellinga, C., Jeppsson, U., Marsili-Libelli, S., Pons, M. N., Spanjers, H. and Vanhooren, H. [1999], Benchmark for evaluating control strategies in wastewater treatment plants, in 'European Control Conference', Karlsruhe, Germany.

[3] Basseville, M. [1998], 'On-board component fault detection and isolation using the statistical local approach', Automatica 34(11), 1391-1415.

[4] Besançon, G. [2000], 'Remarks on nonlinear adaptive observer design', Systems and Control Letters 41(4), 271-280.

[5] Carlos-Hernandez, S., Sanchez, E. and Béteau, J. F. [2009], 'Fuzzy observers for anaerobic WWTP: development and implementation', Control Engineering Practice 17(6), 690-702.

[6] Chen, J. and Patton, R. [1999], Robust model based fault diagnosis for dynamic systems, Kluwer Academic Publishers, Boston, Dordrecht, London.

[7] Henze, M., Grady, C. J., Gujer, W., Marais, G. and Matsuo, T. [1987], Activated sludge model no.1., Technical report, I.A.W.P.R.C. Scientific and Technical Report, London, U.K.

[8] Ichalal, D., Marx, B., Ragot, J. and Maquin, D. [2010], 'State estimation of Takagi-Sugeno systems with unmeasurable premise variables', IET Control Theory \& Applications 4(5), 897-908.

[9] Kenne, G., Ahmed-Ali, T., Lamnabhi-Lagarrigue, F. and Arzande, A. [2008], 'Nonlinear systems time-varying parameter estimation: Application to induction motors', Electric Power Systems Research 78(11), 1881-1888.

[10] Labit, Y., Peaucelle, D. and Henrion, D. [2002], SEDUMI interface 1.02: a tool for solving LMI problems with SEDUMI, in 'IEEE International Symposium on Computer Aided Control System Design'.

[11] Lubenova, V. [1999], 'State adaptive algorithm for simultaneous estimation of time-varying parameters and state variables in aerobic bioprocesses', Bioprocess 21(3), 219-226.

[12] Nagy, A. M., Marx, B., Mourot, G., Schutz, G. and Ragot, J. [2011a], 'Observers design for uncertain Takagi-Sugeno systems with unmeasurable premise variables and unknown inputs. Application to a wastewater treatment plant', Journal of Process Control 21(7), 1105-1114. 
[13] Nagy, A. M., Marx, B., Mourot, G., Schutz, G. and Ragot, J. [2011b], 'State estimation of two-time scale multiple models. Application to wastewater treatment plant', Control Engineering Practice 19(11), 1354-1362.

[14] Nagy, A. M., Mourot, G., Marx, B., Ragot, J. and Schutz, G. [2010], 'Systematic multimodeling methodology applied to an activated sludge reactor model', Industrial Engineering Chemical Research 49(6), 2790-2799.

[15] Nagy Kiss, A. M., Marx, B., Mourot, G., Schultz, G. and Ragot, J. [2010], State estimation of two time-scale multiple models with unmeasurable premise variables. Application to biological reactors, in ' $49^{\text {th }}$ IEEE Conference on Decision and Control', Atlanta, Georgia, USA.

[16] Rajamani, R. and Hedrich, J. [1995], 'Adaptive observer for active automotive suspensions- theory and experiment', IEEE Transactions on Control Systems Technology 3(1), 86-93.

[17] SeDuMi. An open source solver for $L P+S O C P+S D P$, written in Matlab [n.d.]. URL: http://sedumi.ie.lehigh.edu/

[18] Takagi, T. and Sugeno, M. [1985], 'Fuzzy identification of systems and its applications to modeling and control', IEEE Transactions on Systems, Man, and Cybernetics 15(1), 116-132.

[19] Tanaka, K. and Wang, H. [2001], Fuzzy Control Systems Design and Analysis: A Linear Matrix Inequality Approach, John Wiley \& Sons, Inc.

[20] Weijers, S. [2000], Modelling, identification and control of activated sludge plants for nitrogen removal, $\mathrm{PhD}$ thesis, Technische Universiteit Eindhoven, Eindhoven, The Netherlands.

[21] Zhang, Q. [2002], 'Adaptive observer for multiple-input-multiple-output (MIMO) linear time-varying systems', IEEE Transactions on Automatic Control 47(3), 525-529.

[22] Zhang, Q. and Xu, A. [2001], Global adaptive observer for a class of nonlinear systems, in 'Conference on Decision and Control CDC', Orlando, Florida, USA.

[23] Zhou, K. and Khargonekar, P. [1988], 'Robust stabilization of linear systems with norm-bounded time-varying uncertainty', Systems and Control Letters 10(1), 1720. 